\title{
A Lyusternik-Graves Theorem for the Proximal Point Method
}

\author{
Francisco J. Aragón Artacho ${ }^{1}$ and MichaËl GaYdu ${ }^{2}$
}

\begin{abstract}
We consider a generalized version of the proximal point algorithm for solving the perturbed inclusion $y \in T(x)$, where $y$ is a perturbation element near 0 and $T$ is a set-valued mapping acting from a Banach space $X$ to a Banach space $Y$ which is metrically regular around some point point $(\bar{x}, 0)$ in its graph. We study the behavior of the convergent iterates generated by the algorithm and we prove that they inherit the regularity properties of $T$, and vice versa. We analyze the cases when the mapping $T$ is metrically regular and strongly regular.
\end{abstract}

Key words: Proximal point algorithm, generalized equations, perturbations, metric regularity, strong regularity.

AMS 2000 Subject Classification: 49J53, 49J40, 90C48.

\section{Introduction}

The proximal point method finds its origin in the works of Martinet [19] in 1970 for variational inequalities. A few years later Rockafellar [25, 26] thoroughly studied the algorithm, showing its tight relation to multiplier methods. The algorithm aims to find an approximate solution to the equation

$$
0 \in T(x),
$$

\footnotetext{
${ }^{1}$ Department of Statistics and Operations Research, University of Alicante, 03080 Alicante, Spain, francisco.aragon@ua.es. This author is supported by MICINN of Spain, grant MTM2008-06695-C0301 and program "Juan de la Cierva."

${ }^{2}$ LAMIA, Dpt. de Mathématiques, Université Antilles-Guyane, F-97159 Pointe-à-Pitre, Guadeloupe, michael.gaydu@univ-ag.fr. This author is supported by Contract EA4540 (France).
} 
for a given set-valued mapping $T$, typically acting from a Hilbert space to itself. Rockafellar proved, in particular, that the following iterative process

$$
0 \in \lambda_{n}\left(x_{n+1}-x_{n}\right)+T\left(x_{n+1}\right) \text { for } n=0,1,2, \ldots
$$

known as the exact proximal point method (where $\lambda_{n}$ is a sequence of positive numbers and $x_{0} \in X$ is a starting point), provides a sequence $x_{n}$ which is weakly convergent to a solution to (1.1) when $T$ is a maximal monotone operator. The particular case when $T$ is the subdifferential of a lower semicontinuous convex function is of special relevance; here the subproblem (1.2) becomes

$$
x_{n+1}:=\underset{z}{\operatorname{argmin}}\left\{f(z)+\frac{\lambda_{n}}{2}\left\|z-x_{n}\right\|^{2}\right\},
$$

transforming thus the single problem of minimizing a convex function into solving a sequence of problems where the objective function is strongly convex (which improves the convergence properties of some minimization algorithms). On the other hand, the term $\left\|z-x_{n}\right\|^{2}$ forces the next iteration to remain proximal to the previous one, while the parameter $\lambda_{n}$ provides control on this effect.

Subsequently, several authors have studied generalizations or modifications of the proximal point method in order to implement or improve the theoretical algorithm initiated by Rockafellar, together with some inexact versions for the subproblem (1.2); we can cite, for instance, $[13,15,16,17,18,22,28,29,30]$. Notice that allowing approximate solutions in the subproblem (1.2) is crucial in practice; otherwise, solving exactly each of the subproblems might be as difficult as finding a solution to the original inclusion (1.1). Most of the extensive and rapidly growing literature on the subject assumes some sort of maximal monotonicity of the mapping $T$. The reason for this is that it guarantees the uniqueness of solutions to (1.2), having moreover nonexpansivity of the so-called proximal mapping $P_{c}:=(I+c T)^{-1}$ for any $c>0$, i.e.,

$$
\left\|P_{c}(z)-P_{c}\left(z^{\prime}\right)\right\| \leq\left\|z-z^{\prime}\right\| \text { for all } z, z^{\prime},
$$

which is employed to prove the convergence of the sequence $x_{n}$ of solutions of (1.2) to a solution of the original problem (1.1). Without monotonicity everything is more complicated, not only because in some cases we may have multivaluedness or even empty-valuedness in (1.2), but also because the sequence generated by the method might not even converge.

In the present paper we will not assume any type of monotonicity. Instead, the mapping $T$ is required to be either metrically regular or strongly regular around some reference solution (a proper definition of these concepts will be given in the next section). These properties are local, therefore the convergence results will be local as well. Some results in this direction can be found in [22], where Pennanen proves that the strong regularity of the mapping $T$ guarantees the (local) maximal monotonicity of the Yosida regularization $T_{\rho}:=\left(T^{-1}+\rho I\right)^{-1}$ of the mapping $T$ for any $\rho$ bigger than the regularity modulus of $T$, and this property of $T_{\rho}$ ensures the local existence and uniqueness of a proximal point sequence which is linearly convergent to the reference solution, for any initial point close enough to the solution. Other related results can be found in $[1,2]$, where the authors propose a generalization 
of the proximal point method which basically consists in replacing the constants $\lambda_{n}$ by some functions $g_{n}$ which are Lipschitz continuous on some neighborhood of 0 with Lipschitz constants $\lambda_{n}$. This modification of the method allows the mapping $T$ to act between two different spaces $X$ and $Y$, which are assumed to be Banach. More specifically, choose a sequence of Lipschitz continuous function $g_{n}: X \rightarrow Y$ and consider the following algorithm:

$$
0 \in g_{n}\left(x_{n+1}-x_{n}\right)+T\left(x_{n+1}\right) \text { for } n=0,1,2, \ldots
$$

If the Lipschitz constants $\lambda_{n}$ are upper bounded by one over twice the regularity modulus of $T$ around the reference solution, then for any initial point sufficiently close to the solution, there exists a sequence satisfying (1.5) which is linearly convergent to this solution (see [1, Theorem 3.1]). Furthermore, the convergence is superlinear when $\lambda_{n}$ converges to 0 . When $T$ happens to be strongly regular, the sequence is unique (within a certain neighborhood). In [2] the authors prove something more, that for any $y$ close to 0 , if one considers the perturbed problem

$$
y \in T(x),
$$

then under metric regularity there exists a solution to this equation and a proximal point sequence satisfying

$$
y \in g_{n}\left(x_{n+1}-x_{n}\right)+T\left(x_{n+1}\right) \text { for } n=0,1,2, \ldots
$$

which converges (super)linearly to that solution as long as the sequence $\lambda_{n}$ is sufficiently small for all $n$. Similarly, local uniqueness of the sequence is guaranteed under strong regularity.

An interesting mixed approach can be found in [18], where Leventhal assumes both maximal monotonicity and metric subregularity (a weaker form of metric regularity) of the mapping $T$ around some solution $\bar{x}$, and proves the local (super)linear convergence of the algorithm. For the particular case of minimizing a lower semicontinuous convex function $f$ mentioned above, since the subdifferential $\partial f$ is a maximal monotone mapping (see [24, Theorem A]), the linear convergence of the algorithm is then guaranteed under metric subregularity of the subdifferential. On the other hand, in [3] the authors prove that metric subregularity of the subdifferential of $f$ at $(\bar{x}, 0)$ is equivalent to the following quadratic growth condition of the function $f$ : there exist a neighborhood $U$ of $\bar{x}$ and a positive constant $c$ such that

$$
f(x) \geq f(\bar{x})+c d\left(x,(\partial f)^{-1}(0)\right)^{2} \quad \text { whenever } x \in U \text {; }
$$

thus the exact proximal point algorithm (1.3) is linearly convergent when this latter condition holds, and the convergence is superlinear if $\lambda_{n}$ converges to 0 . A characterization for the (strong) regularity of the subdifferential is also given in [3]. One can find a condition for the convergence of the proximal point algorithm somehow similar to (1.8) in [12], cf. Theorem 3.1 .

In the present paper we will consider the exact generalized proximal point algorithm (1.7), as in $[1,2]$. We will follow the same idea from $[4,9]$, where the authors extend the paradigm of the Lyusternik-Graves theorem (see, e.g., [8]) to the framework of a mapping acting from the pair initial point-parameter to the set of convergent Newton sequences associated with 
them. Under some surjectivity assumption, known as ample parameterization, the (strong) metric regularity of the generalized equation is proved to be equivalent to the (strong) metric regularity of the inverse mapping associated with convergent Newton sequences. These results can be understood as some sort of Lyusternik-Graves theorem, where instead of considering a metrically regular mapping which is perturbed by some Lipschitz function, we iteratively perturb a mapping by several Lipschitz functions whose Lipschitz moduli are small enough to preserve, no only the metric regularity of their sum, but the Lipschitzian properties of the mapping that associates to each pair initial iteration-perturbation the set of converging sequences satisfying the algorithm. This can be seen as a sequential implicit function theorem, see [9] and [8, Section 6D] for more details.

Notice that the metric regularity condition on $T$ plus the Lipschitz continuity of $g_{n}$ with appropriately small moduli will somehow substitute the nonexpansivity condition (1.4) which came for granted from the maximal monotonicity assumption, while the strong regularity will now guarantee the (local) uniqueness of the proximal point sequence.

The paper is organized as follows. In Section 2 we present some background material, basically about metric regularity. In Section 3 we give the statement and proof of the main results, which show the equivalence between the metric regularity of $T$ and the Aubin property of the set of convergent proximal point sequences generated by the proximal point method (Theorem 3.3), and the strong regularity of $T$ and the existence of a Lipschitz singlevalued localization of the mapping associated with the convergent sequences (Theorem 3.4). We also present another result, Theorem 3.2, regarding the existence of a (super)linearly convergent proximal point sequence under metric regularity of $T$, which not only improves both [1, Theorem 3.1] and [2, Theorem 3.2], but is also essential for proving the main theorems. Finally, in the same way as in [10,11], it may be interesting to note that such results show in particular (in a certain perspective), that the proximal method is really wellposed since actually the whole proximal iterates of the problem depend continuously on the data of the problem. The results presented are theoretical; we are interested in studying how the regularity properties (and moduli) carry away from the mapping $T$ to the set of convergent proximal point sequences, and vice versa. We believe these properties may have more practical consequences on the implication of different errors in the algortihm, but we shall not go further into this in here.

\section{Background material}

Throughout, $X$ and $Y$ are Banach spaces. We denote a set-valued mapping from $X$ into the subsets of $Y$ by $F: X \rightrightarrows Y$. The graph of $F$ is the set gph $F=\{(x, y) \in X \times Y \mid y \in F(x)\}$, while $F^{-1}$ is the inverse mapping of $F$ defined by $x \in F^{-1}(y) \Leftrightarrow y \in F(x)$. Single-valued mappings, also called functions, are represented by $f: X \rightarrow Y$. The distance from a point $x$ to a set $C$ is denoted by $d(x, C)$; i.e., $d(x, C)=\inf _{y \in C}\|x-y\|$, with the usual convention

$d(x, \emptyset):=\infty$. Recall that $\mathbb{B}_{r}(x)$ stands for the closed ball of radius $r$ centered at $x$. The excess from a set $A$ to a set $B$ is defined by $e(A, B)=\sup _{x \in A} d(x, B)$, with the convention $e(\emptyset, D):=0$ when $D \neq \emptyset$, and $e(\emptyset, \emptyset):=\infty$. We say that a set $C \subset X$ is locally closed around some point $z \in C$ if there exists some constant $\rho>0$ such that the set $C \cap \mathbb{B}_{\rho}(z)$ is closed. 
Finally, $c l_{\infty}(X)$ denotes the linear space of all infinite sequences $\xi=\left\{x_{1}, x_{2}, \ldots, x_{n} \ldots\right\}$ with elements $x_{n} \in X$, for $n=1,2, \ldots$, that are convergent to some point $x \in X$. We endow this set with the supremum norm

$$
\|\xi\|_{\infty}=\sup _{n \geq 1}\left\|x_{n}\right\|
$$

which makes it a linear normed space.

Our study is focused on two key notions: metric regularity and strong metric regularity.

Definition 2.1. A mapping $F: X \rightrightarrows Y$ is said to be metrically regular around $(\bar{x}, \bar{y}) \in \operatorname{gph} F$ if there exist some positive constants $\kappa, a$ and $b$ such that

$$
d\left(x, F^{-1}(y)\right) \leq \kappa d(y, F(x)) \text { for all } x \in \mathbb{B}_{a}(\bar{x}) \text { and } y \in \mathbb{B}_{b}(\bar{y})
$$

The infimum of $\kappa$ over all the combinations $(\kappa, a, b)$ for which $(2.1)$ holds is the regularity modulus and is denoted by $\operatorname{reg} F(\bar{x}, \bar{y})$; the absence of this property is signaled by $\operatorname{reg} F(\bar{x}, \bar{y})=\infty$. Metric regularity of a mapping $F$ around $(\bar{x}, \bar{y})$ is known to be equivalent to the Aubin property of the inverse $F^{-1}$ around $(\bar{y}, \bar{x})$ with the same modulus (see, e.g., [8, Theorem 5A.3] or [21, Theorem 1.49]). Recall that a set-valued mapping $G: Y \rightrightarrows X$ has the Aubin property (or, equivalently, is Lipschitz-like) around $(\bar{y}, \bar{x}) \in \operatorname{gph} G$ if there exist positive constants $\ell, a$ and $b$ such that,

$$
e\left(G(y) \cap \mathbb{B}_{a}(\bar{x}), G\left(y^{\prime}\right)\right) \leq \ell\left\|y-y^{\prime}\right\| \text { for all } y, y^{\prime} \in \mathbb{B}_{b}(\bar{y}) .
$$

A mapping $H: P \times Y \rightrightarrows X$ is said to have the partial Aubin property with respect to $p$ uniformly in $y$ around $((\bar{p}, \bar{y}), \bar{x})$ if $\bar{x} \in H(\bar{p}, \bar{y})$ and there are some positive constants $\ell, a, b$ and $c$ such that

$$
e\left(H(p, y) \cap \mathbb{B}_{a}(\bar{x}), H\left(p^{\prime}, y\right)\right) \leq \ell\left\|p-p^{\prime}\right\| \quad \text { for all } p, p^{\prime} \in \mathbb{B}_{b}(\bar{p}) \text { and } y \in \mathbb{B}_{c}(y)
$$

The infimum of $\ell$ over all such combinations of $(\ell, a, b, c)$ is called the partial Lipschitz modulus of $H$ with respect to $p$ uniformly in $y$ around $((\bar{p}, \bar{y}), \bar{x})$ and is denoted by $\widehat{\operatorname{lip}}_{p} H((\bar{p}, \bar{y}), \bar{x})$. It is easy to see that the Aubin property of $H$ around $((\bar{p}, \bar{y}), \bar{x})$ ensures the partial Aubin properties of $H$ with respect to both $p$ and $y$.

In order to introduce the second regularity property that we will employ, we recall here the notion of graphical localization. We say that a mapping $\tilde{F}: X \rightrightarrows Y$ is a graphical localization of a set-valued mapping $F: X \rightrightarrows Y$ around $(\bar{x}, \bar{y}) \in \operatorname{gph} F$ if there exists some neighborhood $U \times V$ of $(\bar{x}, \bar{y})$ such that the graph of $F$ restricted to $U \times V$ coincides with the graph of $\tilde{F}$; i.e., gph $\tilde{F}=(U \times V) \cap \operatorname{gph} F$.

Definition 2.2. A mapping $F: X \rightrightarrows Y$ is strongly (metrically) regular around $(\bar{x}, \bar{y}) \in$ gph $F$ if the metric regularity condition (2.1) is satisfied by some $(\kappa, a, b)$ and, in addition, the graphical localization $\mathbb{B}_{b}(\bar{y}) \ni y \mapsto F^{-1}(y) \cap \mathbb{B}_{a}(\bar{x})$ is nowhere multivalued.

Strong regularity of $F$ around $(\bar{x}, \bar{y})$ is equivalent to $F^{-1}$ having a Lipschitz continuous single-valued localization around $(\bar{y}, \bar{x})$, see [8, Proposition 3G.1] for details. This property was introduced by Robinson [23] for variational inequalities and it has been widely applied 
afterwards to sensitivity analysis and numerical methods for optimization. For more details on these and other regularity notions and applications to variational problems one can refer to $[7,14,20]$ and the monographs $[5,8,21,27]$.

The next theorem, originally proved in [6] by an iterative process (see also [8, Theorem 5.E2]), has a crucial role for establishing our main results. It generalizes the classical fixed point theorem to a set-valued mapping setting, and states as follows.

Theorem 2.3 (contraction mapping principle for set-valued mappings). Let $(X, d)$ be $a$ complete metric space, and consider a set-valued mapping $\Phi: X \rightrightarrows X$, a point $\bar{x} \in X$, and positive scalars $\alpha$ and $\theta$ such that $\theta<1$, the set $\operatorname{gph} \Phi \cap\left(\mathbb{B}_{\alpha}(\bar{x}) \times \mathbb{B}_{\alpha}(\bar{x})\right)$ is closed, and the following conditions hold:

(i) $d(\bar{x}, \Phi(\bar{x}))<\alpha(1-\theta)$;

(ii) $e\left(\Phi(u) \cap \mathbb{B}_{\alpha}(\bar{x}), \Phi(v)\right) \leq \theta d(u, v)$ for all $u, v \in \mathbb{B}_{\alpha}(\bar{x})$.

Then $\Phi$ has a fixed point in $\mathbb{B}_{\alpha}(\bar{x})$; that is, there exists $x \in \mathbb{B}_{\alpha}(\bar{x})$ such that $x \in \Phi(x)$.

\section{Lipschitzian behavior of proximal iterates}

In this main section we study the Lipschitzian behavior of proximal iterates, i.e., iterates generated by the proximal point method (1.7). In particular, we show that for any perturbation vectors $y$ and $y^{\prime}$ close to 0 , any initial point $x_{0}^{\prime}$ nearby $\bar{x}$, and any convergent sequence $\xi=\left\{x_{1}, x_{2}, \ldots\right\}$ with starting point $x_{0}$ close to $\bar{x}$ and satisfying (1.7), one can find another proximal point sequence $\xi^{\prime}=\left\{x_{1}^{\prime}, x_{2}^{\prime}, \ldots\right\}$ starting from $x_{0}^{\prime}$ and (linearly/superlinearly) convergent to some solution to $y^{\prime} \in T(x)$ which stays at a distance from $\xi$ proportional both to the distance between the perturbations $y$ and $y^{\prime}$, and between the initial points $x_{0}$ and $x_{0}^{\prime}$. Such results may have important implication in the analysis of the effect of various errors, including the errors of approximations of the problem, and show, in particular, how the initial data affect the algorithm at each step.

Define the set-valued mapping $\Gamma: X \times Y \rightrightarrows c l_{\infty}(X)$ as follows

$$
\begin{gathered}
\Gamma:(u, y) \mapsto\left\{\xi=\left\{x_{1}, x_{2}, \ldots\right\} \in c l_{\infty}(X) \mid y \in \bigcap_{n \geq 0}\left(T\left(x_{n+1}\right)+g_{n}\left(x_{n+1}-x_{n}\right)\right)\right. \\
\text { with } \left.x_{0}=u\right\} .
\end{gathered}
$$

For a given $y$ nearby 0 and an initial point $u$, the set $\Gamma(u, y)$ consists of those convergent sequences satisfying (1.7) for all $n$. When $(\bar{x}, 0) \in \operatorname{gph} T$, we denote by $\bar{\xi}$ the constant sequence with all elements equal $\bar{x}$; then, directly from the definition of $\Gamma$, we have $\bar{\xi}=$ $(\bar{x}, \bar{x}, \bar{x}, \ldots) \in \Gamma(\bar{x}, 0)$.

We will use several times the following technical lemma, which is a direct consequence of Theorem 2.3 .

Lemma 3.1. Let $T: X \rightrightarrows Y$ be a set-valued mapping, let $g: X \rightarrow Y$ be a function with $g(0)=0$, and let $\bar{x} \in X$ be such that $0 \in T(\bar{x})$. Pick some positive constants $a, b, c$, $\alpha$, and $\eta$. 
Choose some points $u \in \mathbb{B}_{b}(\bar{x}), v \in \mathbb{B}_{c}(\bar{x}), y \in \mathbb{B}_{\eta}(0)$, and consider the set-valued mapping $\Phi_{u, y}: X \rightrightarrows X$ defined by $\Phi_{u, y}(\cdot):=T^{-1}(y-g(\cdot-u))$. Assume that the next conditions hold:

(i) the above constants satisfy

$$
\kappa \lambda<1, \quad \alpha+b+c \leq a, \quad \text { and } \eta+\lambda(\alpha+b+c) \leq a
$$

(ii) the mapping $T$ is metrically regular around $(\bar{x}, 0)$ with constant $\kappa$ and neighborhoods $\mathbb{B}_{a}(\bar{x})$ and $\mathbb{B}_{a}(0)$;

(iii) the set $\operatorname{gph} T \cap\left(\mathbb{B}_{a}(\bar{x}) \times \mathbb{B}_{a}(0)\right)$ is closed;

(iv) the function $g$ is Lipschitz continuous with constant $\lambda$ on $\mathbb{B}_{a}(0)$;

(v) there is some $y^{\prime} \in T(v)$ such that

$$
\alpha>\frac{\kappa}{1-\kappa \lambda}\left\|y-y^{\prime}-g(v-u)\right\| .
$$

Then $\Phi_{u, y}$ has a fixed point on $\mathbb{B}_{\alpha}(v)$.

Proof. We will first prove that the set gph $\Phi_{u, y} \cap\left(\mathbb{B}_{\alpha}(v) \times \mathbb{B}_{\alpha}(v)\right)$ is closed. Let $\left(z_{n}, w_{n}\right) \in$ $\operatorname{gph} \Phi_{u, y} \cap\left(\mathbb{B}_{\alpha}(v) \times \mathbb{B}_{\alpha}(v)\right)$ be such that $\left(z_{n}, w_{n}\right)$ converges to some point $(z, w) \in X \times X$. Then $\left(w_{n}, y-g\left(z_{n}-u\right)\right) \in \operatorname{gph} T$. Since

$$
\left\|z_{n}-u\right\| \leq\left\|z_{n}-v\right\|+\|v-\bar{x}\|+\|\bar{x}-u\| \leq \alpha+c+b \leq a,
$$

using (i) and (iv), we have

$$
\left\|y-g\left(z_{n}-u\right)\right\| \leq \eta+\lambda(\alpha+b+c) \leq a
$$

On the other hand,

$$
\left\|w_{n}-\bar{x}\right\| \leq\left\|w_{n}-v\right\|+\|v-\bar{x}\| \leq \alpha+c \leq a .
$$

Therefore $\left(w_{n}, y-g\left(z_{n}-u\right)\right) \in \operatorname{gph} T \cap\left(\mathbb{B}_{a}(\bar{x}) \times \mathbb{B}_{a}(0)\right)$, which is closed; whence $(w, y-$ $g(z-u)) \in \operatorname{gph} T$. This implies that $(z, w) \in \operatorname{gph} \Phi_{u, y} \cap\left(\mathbb{B}_{\alpha}(v) \times \mathbb{B}_{\alpha}(v)\right)$ and proves the closedness of this set.

Now we will verify the rest of the assumptions of Theorem 2.3. Observe that $\|v-u\| \leq$ $c+b \leq a$, and thus, thanks to (i) and (iv), we obtain

$$
\|y-g(v-u)\| \leq \eta+\lambda(b+c) \leq a .
$$

Hence, using (ii), (iv), and (v), we get

$$
\begin{aligned}
d\left(v, \Phi_{u, y}(v)\right) & \leq \kappa d(y-g(v-u), T(v)) \leq \kappa\left\|y-y^{\prime}-g(v-u)\right\| \\
& <\alpha(1-\kappa \lambda)
\end{aligned}
$$

On the other hand, for any $z \in \mathbb{B}_{\alpha}(v)$, one has

$$
\|z-\bar{x}\| \leq\|z-v\|+\|v-\bar{x}\| \leq \alpha+c \leq a ;
$$


thus, $\mathbb{B}_{\alpha}(v) \subset \mathbb{B}_{a}(\bar{x})$. For all $w, w^{\prime} \in \mathbb{B}_{\alpha}(v)$, one has

$$
\|w-u\| \leq\|w-v\|+\|v-u\| \leq \alpha+b+c \leq a,
$$

and likewise, $\left\|w^{\prime}-u\right\| \leq a$. Therefore, using again (ii) and (iv), we obtain

$$
\begin{aligned}
e\left(\Phi_{u, y}(w) \cap \mathbb{B}_{\alpha}(v), \Phi_{u, y}\left(w^{\prime}\right)\right) & =\sup _{z \in \Phi_{u, y}(w) \cap \mathbb{B}_{\alpha}(v)} d\left(z, \Phi_{u, y}\left(w^{\prime}\right)\right) \\
& \leq \sup _{z \in \Phi_{u, y}(w) \cap \mathbb{B}_{a}(\bar{x})} d\left(z, T^{-1}\left(y-g\left(w^{\prime}-u\right)\right)\right) \\
& \leq \sup _{z \in T^{-1}(y-g(w-u)) \cap \mathbb{B}_{a}(\bar{x})} \kappa d\left(y-g\left(w^{\prime}-u\right), T(z)\right) \\
& \leq \kappa\left\|g(w-u)-g\left(w^{\prime}-u\right)\right\| \leq \kappa \lambda\left\|w-w^{\prime}\right\| .
\end{aligned}
$$

Thus, all the conditions on Theorem 2.3 are satisfied, whence $\Phi_{u, y}$ has a fixed point on $\mathbb{B}_{\alpha}(v)$, as claimed.

The following result shows that under metric regularity of the mapping $T$ around $(\bar{x}, 0)$, for every pair initial point-perturbation $(u, y)$ close to $(\bar{x}, 0)$ and every solution $x$ to the equation $y \in T(x)$ nearby $\bar{x}$, there exists a sequence satisfying the proximal point method (1.5), starting from $x_{0}=u$, which is linearly convergent to $x$. Moreover, the convergence of this sequence is superlinear when the Lipschitz constants of the functions $g_{n}$ converge to 0 . This result generalizes both [1, Theorem 3.1] and [2, Theorem 3.2], and it will be utilized to prove the main theorem.

Theorem 3.2. Consider a mapping $T: X \rightrightarrows Y$ and any pair $(\bar{x}, 0) \in \operatorname{gph} T$ such that the graph of $T$ is locally closed around $(\bar{x}, 0)$. Choose a sequence of functions $g_{n}: X \rightarrow Y$ with $g_{n}(0)=0$ which are Lipschitz continuous in a neighborhood of 0 , the same for all $n$, with Lipschitz constants $\lambda_{n}$. If $T$ is metrically regular around $(\bar{x}, 0)$ with constant $\kappa$ and $\lambda>\sup _{n} \lambda_{n}$ is such that $2 \kappa \lambda<1$, then there is a positive constant $\delta$ such that, for all $(x, y) \in \operatorname{gph} T \cap\left(\mathbb{B}_{\delta}(\bar{x}) \times \mathbb{B}_{\delta}(0)\right)$ and all $u \in \mathbb{B}_{\delta}(\bar{x})$, there exists a sequence $\xi=\left\{x_{1}, x_{2}, \ldots\right\} \in$ $\Gamma(u, y)$ with

$$
\left\|x_{n+1}-x\right\| \leq \frac{\kappa \lambda_{n}}{1-\kappa \lambda}\left\|x_{n}-x\right\| \text { for all } n .
$$

Therefore, $x_{n}$ is linearly convergent to $x$, and the convergence is superlinear when $\lambda_{n} \downarrow 0$.

Proof. There exists some positive constant $a$ such that:

$$
\begin{gathered}
\text { the set } \operatorname{gph} T \cap\left(\mathbb{B}_{a}(\bar{x}) \times \mathbb{B}_{a}(0)\right) \text { is closed; } \\
d\left(x, T^{-1}(y)\right) \leq \kappa d(y, T(x)) \text { for all }(x, y) \in \mathbb{B}_{a}(\bar{x}) \times \mathbb{B}_{a}(0) \\
\left\|g_{n}(x)-g_{n}\left(x^{\prime}\right)\right\| \leq \lambda_{n}\left\|x-x^{\prime}\right\| \text { for all } x, x^{\prime} \in \mathbb{B}_{a}(0) \text { and } n=0,1,2, \ldots
\end{gathered}
$$

Choose a positive constant $\delta$ such that

$$
6 \delta(1+\lambda) \leq a
$$


$\operatorname{Pick}(x, y) \in \operatorname{gph} T \cap\left(\mathbb{B}_{\delta}(\bar{x}) \times \mathbb{B}_{\delta}(0)\right)$ and $u \in \mathbb{B}_{\delta}(\bar{x})$. By an induction process, we will build a sequence $\xi=\left\{x_{1}, x_{2}, \ldots\right\} \in \Gamma(u, y)$ such that

$$
\left\|x_{n+1}-x\right\| \leq \alpha_{n}:=\frac{\kappa \lambda_{n}}{1-\kappa \lambda}\left\|x_{n}-x\right\| .
$$

If $x=u$ take $\xi:=\{x, x, \ldots, x, \ldots\}$ and we are done. Otherwise define

$$
\alpha_{0}:=\frac{\kappa \lambda_{0}}{1-\kappa \lambda}\|u-x\| \leq\|u-\bar{x}\|+\|\bar{x}-x\| \leq 2 \delta .
$$

By applying Lemma 3.1 to the mapping $\Phi_{0}(\cdot):=T^{-1}\left(y-g_{0}(\cdot-u)\right)$ with $\left(u, v, y, y^{\prime}\right)$ equal to $(u, x, y, y)$ and $(a, b, c, \alpha, \eta)$ equal to $\left(a, \delta, \delta, \alpha_{0}, \delta\right)$, observing that $u \in \mathbb{B}_{\delta}(\bar{x}), x \in \mathbb{B}_{\delta}(\bar{x})$, $y \in \mathbb{B}_{\delta}(0)$, and

$$
\begin{aligned}
& \alpha_{0}+\delta+\delta \leq 4 \delta \leq a, \\
& \delta+\lambda_{0}\left(\alpha_{0}+\delta+\delta\right) \leq \delta(1+4 \lambda) \leq a, \\
& \frac{\kappa}{1-\kappa \lambda_{0}}\left\|y-y-g_{0}(x-u)\right\|<\frac{\kappa \lambda_{0}}{1-\kappa \lambda}\|x-u\|=\alpha_{0} \text {, }
\end{aligned}
$$

we obtain a point $x_{1} \in \mathbb{B}_{\alpha_{0}}(x)$ such that

$$
y \in g_{0}\left(x_{1}-u\right)+T\left(x_{1}\right)
$$

Now assume that, for some $n \geq 1$, the elements in $\left\{x_{1}, \ldots, x_{n}\right\}$ have been defined as proximal iterates satisfying (3.8). If $x_{n}=x$, define $x_{n+1}:=x$ and we are done. Otherwise take $\alpha_{n}=\frac{\kappa \lambda_{n}}{1-\kappa \lambda}\left\|x_{n}-x\right\|$ and consider the mapping $\Phi_{n}(\cdot):=T^{-1}\left(y-g_{n}\left(\cdot-x_{n}\right)\right)$. From (3.8) we get

$$
\left\|x_{n}-\bar{x}\right\| \leq\left\|x_{n}-x\right\|+\|x-\bar{x}\| \leq\|u-x\|+\delta \leq 3 \delta .
$$

Again, we will apply Lemma 3.1 , now to the mapping $\Phi_{n}$, with $\left(u, v, y, y^{\prime}\right)$ equal to $\left(x_{n}, x, y, y\right)$ and $(a, b, c, \alpha, \eta)$ equal to $\left(a, 3 \delta, \delta, \alpha_{n}, \delta\right)$. Conditions (3.2) and (3.3) are satisfied:

$$
\begin{gathered}
\alpha_{n}+3 \delta+\delta \leq 6 \delta \leq a, \\
\delta+\lambda_{n}\left(\alpha_{n}+3 \delta+\delta\right) \leq \delta(1+6 \lambda) \leq a, \\
\frac{\kappa \quad \lambda_{n}}{1-\kappa \lambda_{n}}\left\|y-g_{n}\left(x-x_{n}\right)\right\|<\frac{\kappa \lambda_{n}}{1-\kappa \lambda}\left\|x-x_{n}\right\|=\alpha_{n}
\end{gathered}
$$

then the mapping $\Phi_{n}$ has a fixed point on $\mathbb{B}_{\alpha_{n}}(x)$, that is, there is a point $x_{n+1} \in \Phi_{n}\left(x_{n+1}\right) \cap$ $\mathbb{B}_{\alpha_{n}}(x)$. Thus (3.8) holds, and this completes the inductive definition of the proximal point sequence $\xi=\left\{x_{1}, x_{2}, x_{3}, \ldots\right\}$. Finally, notice that (3.8) implies the linear convergence of the sequence $x_{n}$ to $x$ (superlinear when $\lambda_{n} \downarrow 0$ ); whence $\xi \in \Gamma(u, y)$.

We are now ready to present our main result, which can be viewed as some sort of Lyusternik-Graves-type theorem on the convergent sequences generated by the proximal point algorithm. 
Theorem 3.3. Consider a mapping $T: X \rightrightarrows Y$ and any pair $(\bar{x}, 0) \in \operatorname{gph} T$ such that the graph of $T$ is locally closed around $(\bar{x}, 0)$. Choose a sequence of functions $g_{n}: X \rightarrow Y$ with $g_{n}(0)=0$ which are Lipschitz continuous in a neighborhood of 0 , the same for all $n$, with Lipschitz constants $\lambda_{n}$. The following assertions hold.

(i) If $T$ is metrically regular around $(\bar{x}, 0)$ with $\operatorname{reg} T(\bar{x}, 0) \cdot \sup _{n} \lambda_{n}<1 / 2$, then the mapping $\Gamma$ in (3.1) has the Aubin property around $((\bar{x}, 0), \bar{\xi})$, with

$$
\begin{gathered}
\widehat{\operatorname{lip}}_{u} \Gamma((\bar{x}, 0), \bar{\xi}) \leq \frac{\operatorname{reg} T(\bar{x}, 0) \cdot \sup _{n} \lambda_{n}}{1-\operatorname{reg} T(\bar{x}, 0) \cdot \sup _{n} \lambda_{n}}, \\
\widehat{\operatorname{lip}}_{y} \Gamma((\bar{x}, 0), \bar{\xi}) \leq \frac{\operatorname{reg} T(\bar{x}, 0) \cdot\left(3-4 \operatorname{reg} T(\bar{x}, 0) \cdot \sup _{n} \lambda_{n}\right)}{1-2 \operatorname{reg} T(\bar{x}, 0) \cdot \sup _{n} \lambda_{n}} .
\end{gathered}
$$

(ii) Conversely, if $\Gamma$ has the Aubin property with respect to $y$ uniformly in $u$ around $((\bar{x}, 0), \bar{\xi})$, then $T$ is metrically regular around $(\bar{x}, 0)$. Moreover,

$$
\operatorname{reg} T(\bar{x}, 0) \leq \widehat{\operatorname{lip}}_{y} \Gamma((\bar{x}, 0), \bar{\xi})
$$

Proof. We start with the proof of assertion (i). Let $\kappa>\operatorname{reg} T(\bar{x}, 0)$ and $\lambda>\sup _{n} \lambda_{n}$ be such that $2 \kappa \lambda<1$. Let $\delta>0$ be the constant from Theorem 3.2. There exists some positive constant $a$ such that (3.5), (3.6) and (3.7) hold. Now choose some positive constants $\mu$ and $\eta$ such that

$$
\frac{2 \kappa \eta}{1-2 \kappa \lambda}+3 \mu \leq \delta<a / 6, \quad \eta+\lambda\left(\frac{6 \kappa \eta}{1-2 \kappa \lambda}+6 \mu\right)<a, \quad \text { and } \eta \leq \delta .
$$

Fix $x_{0}, x_{0}^{\prime} \in \mathbb{B}_{\mu}(\bar{x}), y, y^{\prime} \in \mathbb{B}_{\eta}(0)$ with $\left(x_{0}, y\right) \neq\left(x_{0}^{\prime}, y^{\prime}\right)$ and $\xi=\left\{x_{1}, x_{2}, \ldots, x_{n}, \ldots\right\} \in$ $\Gamma\left(x_{0}, y\right) \cap \mathbb{B}_{\mu}(\bar{\xi})$ (if there is no such $\xi$, we are done). Then the sequence $\xi$ is convergent to some point $x \in \mathbb{B}_{\mu}(\bar{x})$.

First, we will build by induction a sequence $\left\{x_{1}^{\prime}, x_{2}^{\prime}, \ldots, x_{k}^{\prime}, \ldots\right\}$ satisfying

$$
y^{\prime} \in T\left(x_{n+1}^{\prime}\right)+g_{n}\left(x_{n+1}^{\prime}-x_{n}^{\prime}\right)
$$

and such that

$$
\left\|x_{n+1}-x_{n+1}^{\prime}\right\| \leq \alpha_{n}:=\frac{\kappa}{1-\kappa \lambda}\left(\left\|y-y^{\prime}\right\|+\lambda\left\|x_{n}-x_{n}^{\prime}\right\|\right)
$$

for $n=0,1,2, \ldots$ Consider the mapping $\Phi_{0}: X \rightrightarrows X$ given by $\Phi_{0}(x):=T^{-1}\left(y^{\prime}-g_{0}\left(x-x_{0}^{\prime}\right)\right)$ for $x \in X$. Observe that

$$
\left\|x_{1}-x_{0}^{\prime}\right\| \leq\left\|x_{1}-\bar{x}\right\|+\left\|\bar{x}-x_{0}^{\prime}\right\| \leq 2 \mu \leq a,
$$

and, similarly, $\left\|x_{1}-x_{0}\right\| \leq 2 \mu \leq a$. We will apply Lemma 3.1 to the mapping $\Phi_{0}$ with $\left(u, v, y, y^{\prime}\right)$ equal to $\left(x_{0}^{\prime}, x_{1}, y^{\prime}, y-g_{0}\left(x_{1}-x_{0}\right)\right)$ and $(a, b, c, \alpha, \eta)$ equal to $\left(a, \mu, \mu, \alpha_{0}, \eta\right)$. As- 
sumptions (3.2) and (3.3) are satisfied:

$$
\begin{gathered}
\alpha_{0}+\mu+\mu \leq \frac{2 \kappa}{1-\kappa \lambda}(\eta+\lambda \mu)+2 \mu \leq \frac{2 \kappa \eta}{1-\kappa \lambda}+4 \mu \leq a, \\
\eta+\lambda\left(\alpha_{0}+\mu+\mu\right) \leq \eta+\lambda\left(\frac{2 \kappa \eta}{1-\kappa \lambda}+4 \mu\right) \leq a \\
\frac{\kappa}{1-\kappa \lambda_{0}}\left\|y^{\prime}-\left(y-g_{0}\left(x_{1}-x_{0}\right)\right)-g_{0}\left(x_{1}-x_{0}^{\prime}\right)\right\|<\alpha_{0} .
\end{gathered}
$$

Hence Lemma 3.1 gives us a point $x_{1}^{\prime} \in \Phi_{0}\left(x_{1}^{\prime}\right) \cap \mathbb{B}_{\alpha_{0}}\left(x_{1}\right)$; that is, $x_{1}^{\prime}$ verifies both (3.13) and (3.14).

Now assume that $\left\{x_{1}^{\prime}, \ldots, x_{k}^{\prime}\right\}$ is already defined for $k \geq 1$ satisfying both (3.13) and (3.14). If $\left(x_{k}, y\right)=\left(x_{k}^{\prime}, y^{\prime}\right)$ then $x_{k+1}^{\prime}:=x_{k+1}$ trivially verifies both (3.13) and (3.14). Otherwise consider the mapping $\Phi_{k}: X \rightrightarrows X$ given by $\Phi_{k}(x):=T^{-1}\left(y^{\prime}-g_{k}\left(x-x_{k}^{\prime}\right)\right)$ for $x \in X$. Notice that

$$
\begin{aligned}
\left\|x_{k}-x_{k}^{\prime}\right\| & \leq \alpha_{k-1}=\frac{\kappa}{1-\kappa \lambda}\left\|y-y^{\prime}\right\|+\frac{\kappa \lambda}{1-\kappa \lambda}\left\|x_{k-1}-x_{k-1}^{\prime}\right\| \leq \ldots \\
& \leq \frac{\kappa}{1-\kappa \lambda}\left\|y-y^{\prime}\right\| \sum_{j=0}^{k-1}\left(\frac{\kappa \lambda}{1-\kappa \lambda}\right)^{j}+\left(\frac{\kappa \lambda}{1-\kappa \lambda}\right)^{k}\left\|x_{0}-x_{0}^{\prime}\right\| \\
& \leq \frac{\kappa}{1-2 \kappa \lambda}\left\|y-y^{\prime}\right\|+\left(\frac{\kappa \lambda}{1-\kappa \lambda}\right)^{k}\left\|x_{0}-x_{0}^{\prime}\right\| \leq \frac{2 \kappa \eta}{1-2 \kappa \lambda}+2 \mu .
\end{aligned}
$$

We will apply Lemma 3.1 to the mapping $\Phi_{k}$ with $\left(u, v, y, y^{\prime}\right)$ equal to $\left(x_{k}^{\prime}, x_{k+1}, y^{\prime}, y-\right.$ $\left.g_{k}\left(x_{k+1}-x_{k}\right)\right)$ and $(a, b, c, \alpha, \eta)$ equal to $\left(a, \frac{2 \kappa \eta}{1-2 \kappa \lambda}+3 \mu, \mu, \alpha_{k}, \eta\right)$. Observe that

$$
\left\|x_{k}^{\prime}-\bar{x}\right\| \leq\left\|x_{k}^{\prime}-x_{k}\right\|+\left\|x_{k}-\bar{x}\right\| \leq \frac{2 \kappa \eta}{1-2 \kappa \lambda}+3 \mu
$$

and since

$$
\alpha_{k} \leq \frac{\kappa}{1-\kappa \lambda}\left[2 \eta+\lambda\left(\frac{2 \kappa \eta}{1-2 \kappa \lambda}+2 \mu\right)\right] \leq \frac{4 \kappa \eta}{1-2 \kappa \lambda}+2 \mu,
$$

we also have that conditions (3.2) and (3.3) hold:

$$
\begin{gathered}
\alpha_{k}+\frac{2 \kappa \eta}{1-2 \kappa \lambda}+3 \mu+\mu \leq \frac{6 \kappa \eta}{1-2 \kappa \lambda}+6 \mu \leq a, \\
\eta+\lambda\left(\alpha_{k}+\frac{2 \kappa \eta}{1-2 \kappa \lambda}+3 \mu+\mu\right) \leq \eta+\lambda\left(\frac{6 \kappa \eta}{1-2 \kappa \lambda}+6 \mu\right) \leq a, \\
\frac{\kappa}{1-\kappa \lambda_{k}}\left\|y^{\prime}-\left(y-g_{k}\left(x_{k+1}-x_{k}\right)\right)-g_{k}\left(x_{k+1}-x_{k}^{\prime}\right)\right\|<\alpha_{k},
\end{gathered}
$$

since

$$
\begin{aligned}
\left\|x_{k+1}-x_{k}^{\prime}\right\| & \leq\left\|x_{k+1}-\bar{x}\right\|+\left\|\bar{x}-x_{k}\right\|+\left\|x_{k}-x_{k}^{\prime}\right\| \\
& \leq \frac{2 \kappa \eta}{1-2 \kappa \lambda}+4 \mu \leq a
\end{aligned}
$$


Then Lemma 3.1 gives us a point $x_{k+1}^{\prime} \in \Phi\left(x_{k+1}^{\prime}\right) \cap \mathbb{B}_{\alpha_{k}}\left(x_{k+1}\right)$; that is, (3.13) and (3.14) hold. This completes the inductive definition of the sequence $\left\{x_{1}^{\prime}, x_{2}^{\prime}, \ldots, x_{n}^{\prime}, \ldots\right\}$.

Choose $\varepsilon>0$ arbitrarily small such that

$$
(\kappa+\varepsilon) 2 \eta+\mu \leq \delta
$$

which is possible thanks to the first inequality in (3.12). The sequence $\xi$ converges to $x$, hence there is some natural number $N_{\varepsilon}$ such that

$$
\left\|x_{n}-x\right\| \leq \varepsilon\left(\left\|y-y^{\prime}\right\|+\left\|x_{0}-x_{0}^{\prime}\right\|\right) \text { for all } n \geq N_{\varepsilon}
$$

and verifying also

$$
\left(\frac{\kappa \lambda}{1-\kappa \lambda}\right)^{N_{\varepsilon}} \leq \varepsilon .
$$

From (3.6) one has

$$
d\left(x, T^{-1}\left(y^{\prime}\right)\right) \leq \kappa d\left(y^{\prime}, T(x)\right) \leq \kappa\left\|y-y^{\prime}\right\|,
$$

since $x \in \mathbb{B}_{\mu}(\bar{x}) \subset \mathbb{B}_{a}(\bar{x})$. Then, there exists $\hat{x}_{\varepsilon} \in T^{-1}\left(y^{\prime}\right)$ such that

$$
\left\|x-\hat{x}_{\varepsilon}\right\| \leq(\kappa+\varepsilon)\left\|y-y^{\prime}\right\| .
$$

We will use Theorem 3.2 to build another proximal point sequence $\xi^{\prime \prime}$ starting on $x_{N_{\varepsilon}}^{\prime}$ and convergent to $\hat{x}_{\varepsilon} \in T^{-1}\left(y^{\prime}\right)$. The required assumptions hold:

$$
\left\|\hat{x}_{\varepsilon}-\bar{x}\right\| \leq\left\|\hat{x}_{\varepsilon}-x\right\|+\|x-\bar{x}\| \leq(\kappa+\varepsilon) 2 \eta+\mu \leq \delta
$$

and also

$$
\left\|x_{N_{\varepsilon}}^{\prime}-\bar{x}\right\| \leq\left\|x_{N_{\varepsilon}}^{\prime}-x_{N_{\varepsilon}}\right\|+\left\|x_{N_{\varepsilon}}-\bar{x}\right\| \leq \frac{2 \kappa \eta}{1-2 \kappa \lambda}+3 \mu \leq \delta
$$

thus there exists $\xi^{\prime \prime}=\left\{x_{1}^{\prime \prime}, x_{2}^{\prime \prime}, \ldots\right\} \in \Gamma\left(x_{N_{\varepsilon}}^{\prime}, y^{\prime}\right)$ with

$$
\left\|x_{n+1}^{\prime \prime}-\hat{x}_{\varepsilon}\right\| \leq \frac{\kappa \lambda_{n}}{1-\kappa \lambda}\left\|x_{n}^{\prime \prime}-\hat{x}_{\varepsilon}\right\| \text { for all } n=1,2, \ldots
$$

Therefore, $x_{n}^{\prime \prime}$ converges to $\hat{x}_{\varepsilon}$. Furthermore, for all $n=1,2, \ldots$, one has

$$
\begin{aligned}
\left\|x_{n}^{\prime \prime}-x_{N_{\varepsilon}+n}\right\| & \leq\left\|x_{n}^{\prime \prime}-\hat{x}_{\varepsilon}\right\|+\left\|\hat{x}_{\varepsilon}-x\right\|+\left\|x-x_{N_{\varepsilon}+n}\right\| \\
& \leq\left\|x_{N_{\varepsilon}}^{\prime}-\hat{x}_{\varepsilon}\right\|+(\kappa+\varepsilon)\left\|y-y^{\prime}\right\|+\varepsilon\left(\left\|y-y^{\prime}\right\|+\left\|x_{0}-x_{0}^{\prime}\right\|\right),
\end{aligned}
$$

because of (3.17) and (3.18). Using also (3.15), one gets

$$
\begin{aligned}
\left\|x_{N_{\varepsilon}}^{\prime}-\hat{x}_{\varepsilon}\right\| & \leq\left\|x_{N_{\varepsilon}}^{\prime}-x_{N_{\varepsilon}}\right\|+\left\|x_{N_{\varepsilon}}-x\right\|+\left\|x-\hat{x}_{\varepsilon}\right\| \\
& \leq\left(\kappa+2 \varepsilon+\frac{\kappa}{1-2 \kappa \lambda}\right)\left\|y-y^{\prime}\right\|+\left[\varepsilon+\left(\frac{\kappa \lambda}{1-\kappa \lambda}\right)^{N_{\varepsilon}}\right]\left\|x_{0}-x_{0}^{\prime}\right\| \\
& \leq\left(2 \varepsilon+\frac{2 \kappa(1-\kappa \lambda)}{1-2 \kappa \lambda}\right)\left\|y-y^{\prime}\right\|+2 \varepsilon\left\|x_{0}-x_{0}^{\prime}\right\|,
\end{aligned}
$$


and hence

$$
\left\|x_{n}^{\prime \prime}-x_{N_{\varepsilon}+n}\right\| \leq\left(4 \varepsilon+\frac{\kappa(3-4 \kappa \lambda)}{1-2 \kappa \lambda}\right)\left\|y-y^{\prime}\right\|+3 \varepsilon\left\|x_{0}-x_{0}^{\prime}\right\|,
$$

for all $n=1,2, \ldots$. Finally, we define a sequence $\xi_{\varepsilon}^{\prime}:=\left\{x_{1}^{\prime}, x_{2}^{\prime}, \ldots, x_{N_{\varepsilon}}^{\prime}, x_{1}^{\prime \prime}, x_{2}^{\prime \prime}, x_{3}^{\prime \prime}, \ldots\right\} \in$ $\Gamma\left(x_{0}^{\prime}, y^{\prime}\right)$. Moreover,

$$
\begin{aligned}
d\left(\xi, \Gamma\left(x_{0}^{\prime}, y^{\prime}\right)\right) & \leq\left\|\xi-\xi_{\varepsilon}^{\prime}\right\|_{\infty}=\max \left\{\max _{1 \leq k \leq N_{\varepsilon}}\left\|x_{k}-x_{k}^{\prime}\right\|, \sup _{n \geq 1}\left\|x_{N_{\varepsilon}+n}-x_{n}^{\prime \prime}\right\|\right\} \\
& \leq\left(4 \varepsilon+\frac{\kappa(3-4 \kappa \lambda)}{1-2 \kappa \lambda}\right)\left\|y-y^{\prime}\right\|+\left(3 \varepsilon+\frac{\kappa \lambda}{1-\kappa \lambda}\right)\left\|x_{0}-x_{0}^{\prime}\right\| .
\end{aligned}
$$

Making $\varepsilon \downarrow 0$, and remembering that $\xi$ was arbitrarily chosen in $\Gamma\left(x_{0}, y\right) \cap \mathbb{B}_{\mu}(\bar{\xi})$, this yields

$$
e\left(\Gamma\left(x_{0}, y\right) \cap \mathbb{B}_{\mu}(\bar{\xi}), \Gamma\left(x_{0}^{\prime}, y^{\prime}\right)\right) \leq \frac{\kappa(3-4 \kappa \lambda)}{1-2 \kappa \lambda}\left\|y-y^{\prime}\right\|+\frac{\kappa \lambda}{1-\kappa \lambda}\left\|x_{0}-x_{0}^{\prime}\right\| .
$$

This implies the Aubin property of $\Gamma$ around $((\bar{x}, 0), \bar{\xi})$ with the bounds $(3.9)$ and (3.10) for the partial moduli, and concludes the first part of the proof.

Conversely, in order to prove assertion (ii), suppose that $\Gamma$ has the Aubin property with respect to $y$ uniformly in $u$ around $((\bar{x}, 0), \bar{\xi})$ with constant $\kappa$. Then, there is some positive constant $a$ such that

$$
e\left(\Gamma(u, y) \cap \mathbb{B}_{a}(\bar{\xi}), \Gamma\left(u, y^{\prime}\right)\right) \leq \kappa\left\|y-y^{\prime}\right\| \quad \text { whenever } y, y^{\prime} \in \mathbb{B}_{a}(0) \text { and } u \in \mathbb{B}_{a}(\bar{x}) .
$$

We will show that $T^{-1}$ has the Aubin property around $(0, \bar{x})$. Pick $y, y^{\prime} \in \mathbb{B}_{a}(0)$ and $x \in$ $T^{-1}(y) \cap \mathbb{B}_{a}(\bar{x})$. Consider $\xi:=\{x, x, \ldots\}$, then clearly $\xi \in \Gamma(x, y) \cap \mathbb{B}_{a}(\bar{\xi})$; whence

$$
d\left(\xi, \Gamma\left(x, y^{\prime}\right)\right) \leq \kappa\left\|y-y^{\prime}\right\| .
$$

For every $\varepsilon>0$ there exists $\xi^{\varepsilon} \in \Gamma\left(x, y^{\prime}\right)$ such that $\left\|\xi-\xi^{\varepsilon}\right\|_{\infty} \leq(\kappa+\varepsilon)\left\|y-y^{\prime}\right\|$, with $\xi^{\varepsilon}=\left\{x_{1}^{\varepsilon}, x_{2}^{\varepsilon}, \ldots\right\}$ and $x^{\varepsilon}:=\lim _{n \rightarrow \infty} x_{n}^{\varepsilon}$. Keeping in mind the local closedness of $\operatorname{gph} T$ around $(\bar{x}, 0)$ and the Lipschitz continuity of $g_{n}$ around 0 , we have $y^{\prime} \in T\left(x^{\varepsilon}\right)$. Finally, observe that, for any $n$, one has

$$
\begin{aligned}
d\left(x, T^{-1}\left(y^{\prime}\right)\right) & \leq\left\|x-x^{\varepsilon}\right\| \leq\left\|x-x_{n}^{\varepsilon}\right\|+\left\|x_{n}^{\varepsilon}-x^{\varepsilon}\right\| \\
& \leq\left\|\xi-\xi^{\varepsilon}\right\|_{\infty}+\left\|x_{n}^{\varepsilon}-x^{\varepsilon}\right\| \\
& \leq(\kappa+\varepsilon)\left\|y-y^{\prime}\right\|+\left\|x_{n}^{\varepsilon}-x^{\varepsilon}\right\| .
\end{aligned}
$$

Passing to the limit as $n \rightarrow \infty$ in the previous chain of inequalities we obtain

$$
d\left(x, T^{-1}\left(y^{\prime}\right)\right) \leq(\kappa+\varepsilon)\left\|y-y^{\prime}\right\| .
$$

This proves the Aubin property of $T^{-1}$ with constant $\kappa+\varepsilon$ around $(0, \bar{x})$, since $x$ was arbitrarily chosen in $T^{-1}(y) \cap \mathbb{B}_{a}(\bar{x})$; thus, $T$ is metrically regular around $(\bar{x}, 0)$ with this same constant. Taking the limit as $\varepsilon$ goes to 0 , and having in mind that $\kappa$ can be chosen arbitrarily close to $\widehat{\operatorname{lip}}_{y} \Gamma((\bar{x}, 0), \bar{\xi})$, we get $(3.11)$. 
Next we will prove that under strong regularity of $T$, the proximal point iteration is uniquely defined within a neighborhood of $\bar{x}$ for all pair initial point-parameter nearby $(\bar{x}, 0)$; and not only this, as in [2, Theorem 3.3], but also that this single-valued graphical localization of the mapping $\Gamma$ is Lipschitzian. Thus, in this case, it is no longer essential to restrict the mapping $\Gamma$ to sequences which are convergent, since locally, there exists only one proximal sequence which will be convergent to the locally unique solution to (1.6). Furthermore, thanks to the local uniqueness of the sequence, the bound (3.10) can be improved.

Theorem 3.4. Under the same assumptions of Theorem 3.3 one has:

(i) If the mapping $T$ is strongly regular around $(\bar{x}, 0)$ with $\operatorname{reg} T(\bar{x}, 0) \cdot \sup _{n} \lambda_{n}<1 / 2$, then the mapping $\Gamma$ in (3.1) has a Lipschitz single-valued localization $\gamma$ around $((\bar{x}, 0), \bar{\xi})$ whose partial Lipschitz moduli are bounded by

$$
\begin{aligned}
& \widehat{\operatorname{lip}}_{u} \gamma(\bar{x}, 0) \leq \frac{\operatorname{reg} T(\bar{x}, 0) \cdot \sup _{n} \lambda_{n}}{1-\operatorname{reg} T(\bar{x}, 0) \cdot \sup _{n} \lambda_{n}} \\
& \widehat{\operatorname{lip}}_{y} \gamma(\bar{x}, 0) \leq \frac{\operatorname{reg} T(\bar{x}, 0)}{1-\operatorname{reg} T(\bar{x}, 0) \cdot \sup _{n} \lambda_{n}}
\end{aligned}
$$

Moreover, for every $(u, y)$ close to $(\bar{x}, 0), \gamma(u, y)$ is a linearly convergent sequence to a locally unique solution to (1.6), and furthermore, the convergence is superlinear when $\lambda_{n} \downarrow 0$.

(ii) Conversely, if the mapping $\Gamma$ has a Lipschitz single-valued localization around $((\bar{x}, 0), \bar{\xi})$ satisfying

$$
\widehat{\operatorname{lip}}_{u} \Gamma((\bar{x}, 0), \bar{\xi})<1
$$

then $T$ is strongly regular around $(\bar{x}, 0)$ with regularity modulus bounded by (3.11).

Proof. In order to prove assertion (i), assume that $T$ is strongly regular $\operatorname{around}(\bar{x}, 0)$ with constant $\kappa$ such that $\kappa \cdot \sup _{n} \lambda_{n}<1 / 2$. Then there are positive constants $\alpha$ and $\beta$ such that the mapping

$$
\mathbb{B}_{\beta}(0) \ni y \mapsto T^{-1}(y) \cap \mathbb{B}_{\alpha}(\bar{x})
$$

is a Lipschitz continuous function with constant $\kappa$. Further, let $\mu$ be a positive constant such that $g_{n}$ is Lipschitz continuous with constant $\lambda_{n}$ on $\mathbb{B}_{\mu}(0)$ for all $n$. Next take some positive constants $a$ and $b$ such that

$$
a \leq \min \{\alpha, \mu / 2\} \text { and } b+2 a \sup _{n} \lambda_{n} \leq \beta
$$

From Theorem 3.3(i), $\Gamma$ has the Aubin property around $((\bar{x}, 0), \bar{\xi})$; hence, we need to show that $\Gamma$ has a graphical localization around $((\bar{x}, 0), \bar{\xi})$ which is not multivalued. Pick $(u, y) \in$ $\mathbb{B}_{a}(\bar{x}) \times \mathbb{B}_{b}(0)$, and suppose there exist two different sequences $\xi=\left\{x_{1}, x_{2}, \ldots, x_{n}, \ldots\right\}$ and $\xi^{\prime}=\left\{x_{1}^{\prime}, x_{2}^{\prime}, \ldots, x_{n}^{\prime}, \ldots\right\}$, both of them in $\Gamma(u, y) \cap \mathbb{B}_{a}(\bar{\xi})$. Denote by $N$ the first index such that $x_{N} \neq x_{N}^{\prime}$. Observe that

$$
\left\|y-g_{N}\left(x_{N+1}-x_{N}\right)\right\| \leq\|y\|+\lambda_{N}\left\|x_{N+1}-x_{N}\right\| \leq b+2 a \lambda_{N} \leq \beta
$$


thus, $T^{-1}\left(y-g_{N-1}\left(x_{N}-x_{N-1}\right)\right) \cap \mathbb{B}_{\alpha}(\bar{x})=\left\{x_{N}\right\}$, and similarly, $T^{-1}\left(y-g_{N-1}\left(x_{N}^{\prime}-x_{N-1}^{\prime}\right)\right) \cap$ $\mathbb{B}_{\alpha}(\bar{x})=\left\{x_{N}^{\prime}\right\}$. Then

$$
\begin{aligned}
\left\|x_{N}-x_{N}^{\prime}\right\| & \leq \kappa\left\|y-g_{N-1}\left(x_{N}-x_{N-1}\right)-\left(y-g_{N-1}\left(x_{N}^{\prime}-x_{N-1}^{\prime}\right)\right)\right\| \\
& \leq \kappa \lambda_{N-1}\left(\left\|x_{N}-x_{N}^{\prime}\right\|+\left\|x_{N-1}-x_{N-1}^{\prime}\right\|\right) \\
& <\left\|x_{N}-x_{N}^{\prime}\right\|
\end{aligned}
$$

since $\kappa \lambda_{N-1}<1, x_{N-1}=x_{N-1}^{\prime}$, and $x_{N} \neq x_{N}^{\prime}$; that is, we obtain a contradiction. The bound (3.19) directly follows from (3.9). To prove (3.20) we just need to observe that both sequences $\left\{x_{1}^{\prime}, x_{2}^{\prime}, \ldots, x_{k}^{\prime}, \ldots\right\}$ and $\left\{x_{1}^{\prime \prime}, x_{2}^{\prime \prime}, \ldots, x_{k}^{\prime \prime}, \ldots\right\}$ in the proof of Theorem 3.3(i) can be made arbitrarily close to $\bar{x}$, and hence one must have

$$
\left\{x_{1}^{\prime \prime}, x_{2}^{\prime \prime}, \ldots, x_{k}^{\prime \prime}, \ldots\right\}=\left\{x_{N_{\varepsilon}+1}^{\prime}, x_{N_{\varepsilon}+2}^{\prime}, \ldots, x_{N_{\varepsilon}+k}^{\prime}, \ldots\right\}
$$

otherwise we would obtain a contradiction, as shown above. Thus, the bound (3.20) comes from (3.14). The last assertion follows from Theorem 3.2.

Conversely, to prove (ii), suppose that $\Gamma$ has a Lipschitz single-valued localization $\gamma$ around $((\bar{x}, 0), \bar{\xi})$. Then, there exist some positive constants $a$ and $b$ such that $(u, y) \mapsto$ $\gamma(u, y):=\Gamma(u, y) \cap \mathbb{B}_{b}(\bar{\xi})$ is a function defined on $\mathbb{B}_{a}(\bar{x}) \times \mathbb{B}_{a}(0)$. From Theorem 3.3(ii) we know that $T$ is metrically regular around $(\bar{x}, 0)$; thus, it remains to show that $T^{-1}$ is locally nowhere multivalued. Because of (3.21), we can choose some positive constants $c$ and $\ell<1$ such that $\gamma$ is Lipschitz continuous with respect to $u$ uniformly in $y$ on $\mathbb{B}_{c}(\bar{x}) \times \mathbb{B}_{c}(0)$ with Lipschitz constant $\ell$. Let $\alpha:=\min \{a, b, c\}$ and $\beta:=\min \{a, c\}$, and pick some points $y \in \mathbb{B}_{\beta}(0)$ and $x, x^{\prime} \in T^{-1}(y) \cap \mathbb{B}_{\alpha}(\bar{x})$. Define two sequences $\xi:=\{x, x \ldots, x, \ldots\} \in \Gamma(x, y) \cap$ $\mathbb{B}_{b}(\bar{\xi})=\gamma(x, y)$ and $\xi^{\prime}:=\left\{x^{\prime}, x^{\prime} \ldots, x^{\prime}, \ldots\right\} \in \Gamma\left(x^{\prime}, y\right) \cap \mathbb{B}_{b}(\bar{\xi})=\gamma\left(x^{\prime}, y\right)$. Then, one has

$$
\left\|x-x^{\prime}\right\|=\left\|\xi-\xi^{\prime}\right\|_{\infty}=\left\|\gamma(x, y)-\gamma\left(x^{\prime}, y\right)\right\|_{\infty} \leq \ell\left\|x-x^{\prime}\right\|,
$$

which implies $x=x^{\prime}$, since $\ell<1$, and we are done.

Remark 3.5. It is interesting to notice that the following condition

$$
\widehat{\operatorname{lip}}_{y} \Gamma((\bar{x}, 0), \bar{\xi}) \cdot \sup _{n} \lambda_{n}<1 / 2
$$

implies in fact (3.21). Indeed, the assumptions on Theorem 3.3(ii) are satisfied; thus,

$$
\operatorname{reg} T(\bar{x}, 0) \cdot \sup _{n} \lambda_{n} \leq \widehat{\operatorname{lip}}_{y} \Gamma((\bar{x}, 0), \bar{\xi}) \cdot \sup _{n} \lambda_{n}<1 / 2 .
$$

Hence, Theorem 3.3(i) can be applied, and from (3.9) one gets

$$
\widehat{\operatorname{lip}}_{u} \Gamma((\bar{x}, 0), \bar{\xi}) \leq \frac{\operatorname{reg} T(\bar{x}, 0) \cdot \sup _{n} \lambda_{n}}{1-\operatorname{reg} T(\bar{x}, 0) \cdot \sup _{n} \lambda_{n}} \leq \frac{\widehat{\operatorname{lip}}_{y} \Gamma((\bar{x}, 0), \bar{\xi}) \cdot \sup _{n} \lambda_{n}}{1-\widehat{\operatorname{lip}}_{y} \Gamma((\bar{x}, 0), \bar{\xi}) \cdot \sup _{n} \lambda_{n}}<1
$$




\section{References}

[1] F. J. Aragón Artacho, A. L. Dontchev and M. H. Geoffroy, Convergence of the Proximal Point Method for Metrically Regular Mappings, ESAIM Proc. 17 (2007), $1-8$.

[2] F. J. Aragón Artacho and M. H. Geoffroy, Uniformity and inexact version of a proximal method for metrically regular mappings, J. Math. Anal. Appl. 335 (2007), no. $1,168-183$.

[3] F. J. Aragón Artacho and M. H. Geoffroy, Characterization of metric regularity of subdifferentials, J. Convex Anal. 15 (2008), no. 2, 365-380.

[4] F. J. Aragón Artacho, A. L. Dontchev, M. Gaydu, M. H. Geoffroy and V. M. Veliov, Metric regularity of Newton's iteration, SIAM J. Control Optim. 49 (2011), no. 2, 339-362.

[5] J. P. Aubin and H. Frankowska, Set-valued Analysis, Birkhäuser, Boston, 1990.

[6] A. L. Dontchev and W. W. Hager, An inverse mapping theorem for set-valued maps, Proc. Amer. Math. Soc. 121 (1994), no. 2, 481-489.

[7] A. L. Dontchev and R. T. Rockafellar, Regularity and conditioning of solution mappings in variational analysis, Set-Valued Anal. 12 (2004), no. 1-2, 79-109.

[8] A. L. Dontchev And R. T. Rockafellar, Implicit functions and solution mappings. A view from variational analysis, Springer Mathematics Monographs, Dordrecht, 2009.

[9] A. L. Dontchev and R. T. Rockafellar, Newton's method for generalized equations: a sequential implicit function theorem, Math. Program. 123 (2010), no. 1, Ser. B, $139-159$.

[10] M. GAYDU, Stability properties of Tikhonov regularization for nonmonotone inclusions, to appear in J. Global Optim.

[11] M. H. Geoffroy, Stability of Mann's iterates under metric regularity, Appl. Math. Comput. 215 (2009), no. 2, 686-694.

[12] W. W. Hager and H. Zhang, Self-adaptive inexact proximal point methods, Comput. Optim. Appl. 39 (2008), no. 2, 161-181.

[13] C. Humes And P. Silva, Inexact proximal point algorithms and descent methods in optimization, Optim. Eng. 6 (2005), no. 2, 257-271.

[14] A. D. Ioffe, Metric regularity and subdifferential calculus. (Russian) Uspekhi Mat. Nauk 55 (2000), no. 3(333), 103-162; translation in Russian Math. Surveys 55 (2000), no. $3,501-558$. 
[15] A. N. Iusem, T. Pennanen And B. F. Svaiter, Inexact variants of the proximal point algorithm without monotonicity, SIAM J. Optim. 13 (2003), no. 4, 1080-1097.

[16] A. Kaplan and R. Tichatschke, Proximal point methods and nonconvex optimization, J. Global Optim. 13 (1998), no. 4, 389-406.

[17] G. Marino and H.-K. Xu, Convergence of generalized proximal point algorithms, Commun. Pure Appl. Anal. 3 (2004), no. 4, 791-808

[18] D. Leventhal, Metric subregularity and the proximal point method, J. Math. Anal. Appl. 360 (2009), no. 2, 681-688.

[19] B. Martinet, Régularisation d'inéquations variationnelles par approximations successives, Rev. Française d'Informat. Recherche Opérationnelle 4 (1970), Ser. R-3, 154-158.

[20] B. S. Mordukhovich, Complete characterization of openness, metric regularity, and Lipschitzian properties of multifunctions, Trans. Amer. Math. Soc. 340 (1993), no. 1, $1-35$.

[21] B. S. Mordukhovich, Variational analysis and generalized differentiation I: Basic theory, Vol. 330, Springer-Verlag, Berlin, 2006.

[22] T. Pennanen, Local convergence of the proximal point algorithm and multiplier methods without monotonicity, Math. Oper. Res., 27 (2002) 170-191.

[23] S. M. Robinson, Strongly regular generalized equations, Math. Oper. Res. 5 (1980), 43-62.

[24] R. T. Rockafellar, On the maximal monotonicity of subdifferential mappings, Pacific J. Math. 33 (1970), 209-216.

[25] R. T. Rockafellar, Monotone operators and the proximal point algorithm, SIAM J. Control Optim. 14 (1976), no. 5, 877-898.

[26] R. T. Rockafellar, Augmented Lagrangians and applications of the proximal point algorithm in convex programming, Math. Oper. Res. 1 (1976), no. 2, 97-116.

[27] R. T. Rockafellar and R. J.-B. Wets, Variational Analysis, Springer-Verlag, Berlin, 1997.

[28] M. V. Solodov And B. F. Svaiter, A hybrid projection-proximal point algorithm, J. Convex Anal. 6 (1999), no. 1, 59-70.

[29] M. V. Solodov and B. F. Svaiter, A unified framework for some inexact proximal point algorithms, Numer. Funct. Anal. Optim. 22 (2001), no. 7-8, 1013-1035.

[30] Z. Yang And B. He, A relaxed approximate proximal point algorithm, Ann. Oper. Res. 133 (2005), 119-125. 